\title{
Desarrollo Ontogénico de la Columna Vertebral y del Esqueleto Caudal en Larvas de Rhamdia quelen en Condiciones de Larvicultura Intensiva
}

\author{
Ontogenic Development of the Vertebral Column and Caudal Skeleton \\ in Rhamdia quelen Larvae in Larviculture Intensive System
}

*David Roque Hernández; ** Jorge Rafael Casciotta; *Juan José Santinón; "Sebastián Sánchez \& "Hugo Alberto Domitrovic

HERNÁNDEZ, D. R.; CASCIOTTA, J. R.; SANTINÓN, J. J.; SÁNCHEZ, S. \& DOMITROVIC, H. A. Desarrollo ontogénico de la columna vertebral y del esqueleto caudal en larvas de Rhamdia quelen en condiciones de larvicultura intensiva. Int. J. Morphol., 30(4):1520-1525, 2012.

RESUMEN: Se analiza por primera vez el desarrollo osteológico de la columna vertebral y del esqueleto caudal en larvas de Rhamdia quelen mantenidas bajo condiciones controladas y con alimentación artificial. Entre los días 1 a 20 posteriores a la eclosión (dpe) se muestrearon a intervalos regulares 400 larvas, que se colorearon y transparentaron siguiendo la técnica de Taylor \& Van Dike. En los primeros días de vida, las larvas presentan una notocorda recta en toda su longitud. El inicio de la formación de la columna vertebral fue observado a los 7 dpe con la aparición de las espinas neurales y hemales, y entre los 9 y 10 dpe para las tres primeras vértebras. La osificación de la columna vertebral ocurrió en sentido cráneo-caudal y fue completa a los 20 dpe. El esqueleto caudal inicia su desarrollo entre los 3 y 4 dpe, con la formación de los hipurales $(\mathrm{H})$, parahipural $(\mathrm{PH})$ y los radios caudales. Los $\mathrm{H} 1$ al 4 se formaron como primordios cartilaginosos en la cara ventral de la porción distal de la notocorda, mientras que posteriormente lo hicieron el H 5 y el PH en el extremo distal y proximal del esqueleto caudal, respectivamente. El complejo centro ural (CCU) se osificó inicialmente en dos partes, una anterior que resulta de la combinación del centro preural 1 y el ural 1 y una posterior con el ural 2. Los primeros radios de la aleta caudal aparecieron en larvas en flexión, mientras que después de los 13 dpe, todos los ejemplares presentaron los radios principales osificados (ocho en la parte superior y ocho en la inferior). El desarrollo de la columna vertebral y del esqueleto caudal en $R$. quelen muestra patrones similares a los descriptos en otros peces teleósteos aunque con algunas particularidades que lo diferencian de los grupos relacionados.

PALABRAS CLAVE: Rhamdia quelen; Larvicultura; Desarrollo osteológico.

\section{INTRODUCTION}

Entre los numerosos estudios realizados para verificar los patrones de desarrollo osteológico en larvas de peces, se observa que la mayoría de ellos proveen información básica que puede ser utilizada para la reconstrucción filogenética (Dunn, 1983), siendo además fundamental para la identificación precisa del momento donde se produce el desarrollo anormal bajo condiciones de cultivo (Gavaia et al., 2002).

Asimismo, el conocimiento de los aspectos morfológicos del desarrollo esquelético, aporta una valiosa información para comprender y prevenir de manera temprana las incidencias de malformaciones en los peces cultivados (Coban et al., 2009). Tales alteraciones pueden estar asociadas, entre otros factores, con la genética (Castro et al., 2008), con condiciones ambientales (Sfakianakis et al., 2006) o a la nutrición (Cahu et al., 2003; Lall \& LewisMcCrea, 2007), representando un problema importante en la piscicultura (Castro et al.).

$R$. quelen es un pez de importancia comercial tanto en Argentina como en Brasil, criado principalmente bajo sistemas de producción semi-intensivos e intensivos (Baldisserotto \& Radünz Neto, 2004; Luchini \& Panné Huidobro, 2008). En los últimos años se observó un incremento en su producción, convirtiéndose en la especie autóctona más cultivada en la región sur de Brasil (Silveira \& Müller, 2010).

\footnotetext{
* Instituto de Ictiología del Nordeste, Facultad de Ciencias Veterinarias, Universidad Nacional del Nordeste, Argentina.

** Universidad Nacional de La Plata, Buenos Aires, Argentina.
} 
En este estudio, se describe por primera vez el desarrollo osteológico de la columna vertebral y del esqueleto caudal en larvas de $R$. quelen mantenidas bajo condiciones controladas y con alimentación artificial.

\section{MATERIAL Y MÉTODO}

El presente trabajo fue realizado en las instalaciones de piscicultura experimental del Instituto de Ictiología del Nordeste (INICNE) de la Facultad de Ciencias VeterinariasUNNE (Corrientes, Argentina). Las larvas de R. quelen fueron obtenidas mediante cruzamientos entre ejemplares adultos del plantel de reproductores del INICNE por inducción artificial con extracto de hipófisis de Prochilodus lineatus. Una vez nacidas las larvas se colocaron en un acuario de larvicultura a una densidad de 30 larvas L-1 y fueron alimentadas dos veces al día con una microdieta elaborada según Hernández et al. (2009). Durante el período de muestreo la calidad del agua del acuario se mantuvo con promedios de $26,7^{\circ} \mathrm{C}$, pH 7,0, 6,0 mg L-1 de oxígeno disuelto, y conductividad de $129,43 \mathrm{mS} \mathrm{cm}-1$.

Un total de 400 larvas fueron muestreadas a intervalos regulares entre el 1 al 20 día posteriores a la eclosión (dpe). Las muestras se fijaron en formol al 10\% y luego se colorearon y transparentaron siguiendo la técnica de Taylor \& Van Dyke. En el recuento total de las vértebras precaudales se incluyeron a las cuatro correspondientes al aparato de Weber.

Las microfotografías de las larvas en preflexión fueron tomadas con microscopio Leica DM500 con cámara digital Leica ICC50, mientras que las larvas en flexión y postflexión fueron registradas con lupa binocular Kyowa Optical modelo SDZ con cámara digital Sony.

\section{RESULTADOS}

Desarrollo de la Columna vertebral. En los primeros días de vida las larvas carecen de material cartilaginoso u óseo, presentando una notocorda recta en toda su longitud (Figs. 1a). El desarrollo completo de la columna vertebral se alcanza a los 20 dpe cuando la misma se observó constituida por los cuerpos vertebrales (CV), los procesos neurales y hemales y las costillas. El cuerpo vertebral inició el proceso de osificación como hueso membranoso, mientras que los elementos restantes consistieron en huesos cartilaginosos.

Los primeros elementos en formarse en la columna vertebral durante los primeros estadíos larvario fueron los procesos neurales y hemales. Las espinas neurales se hicieron visibles como un par de prominencias cartilaginosas en el tercio anterior y dorsal de la notocorda entre los 7 y 10 dpe. Seguidamente, en la región ventral de la notocorda aparecieron las espinas hemales. El crecimiento de estas estructuras presentó una dirección progresiva hacia posterior para el caso de las espinas neurales y anterior y posterior para las espinas hemales. El inicio de la osificación fue registrado entre los 15 a 17 dpe.

Los primeros CV en manifestar una leve osificación fueron correspondientes a las tres primeras vértebras entre los 9 y 10 dpe. Posteriormente lo hicieron las restantes en sentido cráneo-caudal. En cada CV la osificación se inició desde la periferia hacia el centro. La completa osificación de la columna vertebral ocurrió a los 20 dpe, encontrándose 15 vértebras precaudales y 27 caudales (Fig. 1g).

Desarrollo del Esqueleto Caudal. El esqueleto caudal inicia su desarrollo entre los 4 y 5 dpe, con la formación de los hipurales $(\mathrm{H})$, parahipural $(\mathrm{PH})$ y los radios caudales (Fig. 1b). En este período la notocorda comienza a tener una leve flexión. Los H 1, 2, 3 y 4 se formaron como primordios cartilaginosos relacionados a la cara ventral del extremo distal de la notocorda (Fig. 1c-d). Posteriormente, los $\mathrm{H} 1$ y 2 se fusionaron formando una gran placa romboidal. El último en formarse fue el $\mathrm{H} 5$, ventral al extremo distal de la notocorda. Entre los 4 y 5 dpe, se evidenció una estructura cartilaginosa correspondiente al elemento $\mathrm{PH}$.

Los primeros radios de la aleta caudal aparecen en una larva en flexión en correspondencia con los H 3 y 4 , mientras que después de los 13 dpe se pudo observar la completa formación de los radios principales, siendo éstos segmentados y simples. Así, a los 20 dpe se registraron 16 radios principales, de los cuales 8 se encuentran en el lóbulo dorsal asociados a los $\mathrm{H} 3-5$, y los 8 restantes en el lóbulo ventral asociados a los H 1, 2 y PH (Fig. 1d-e). Hasta ese momento, solamente algunos radios principales poseían ramificaciones. Además, se observaron de 11 a 16 radios secundarios simples en la porción dorsal y de 14 a 18 en la porción ventral. En estas estructuras se observó una osificación intensa a los 20 dpe.

El complejo centro ural (CCU) se osificó inicialmente en dos partes, una anterior que resulta de la combinación del centro preural 1 y el ural 1 y una posterior correspondiente al ural 2 (Fig. 1f). El epural se hizo evidente como un único elemento cartilaginoso distalmente a los arcos neurales, mientras el pleurostilo se formó posteriormente al CCU, encontrándose completamente osificado a los 20 dpe. 

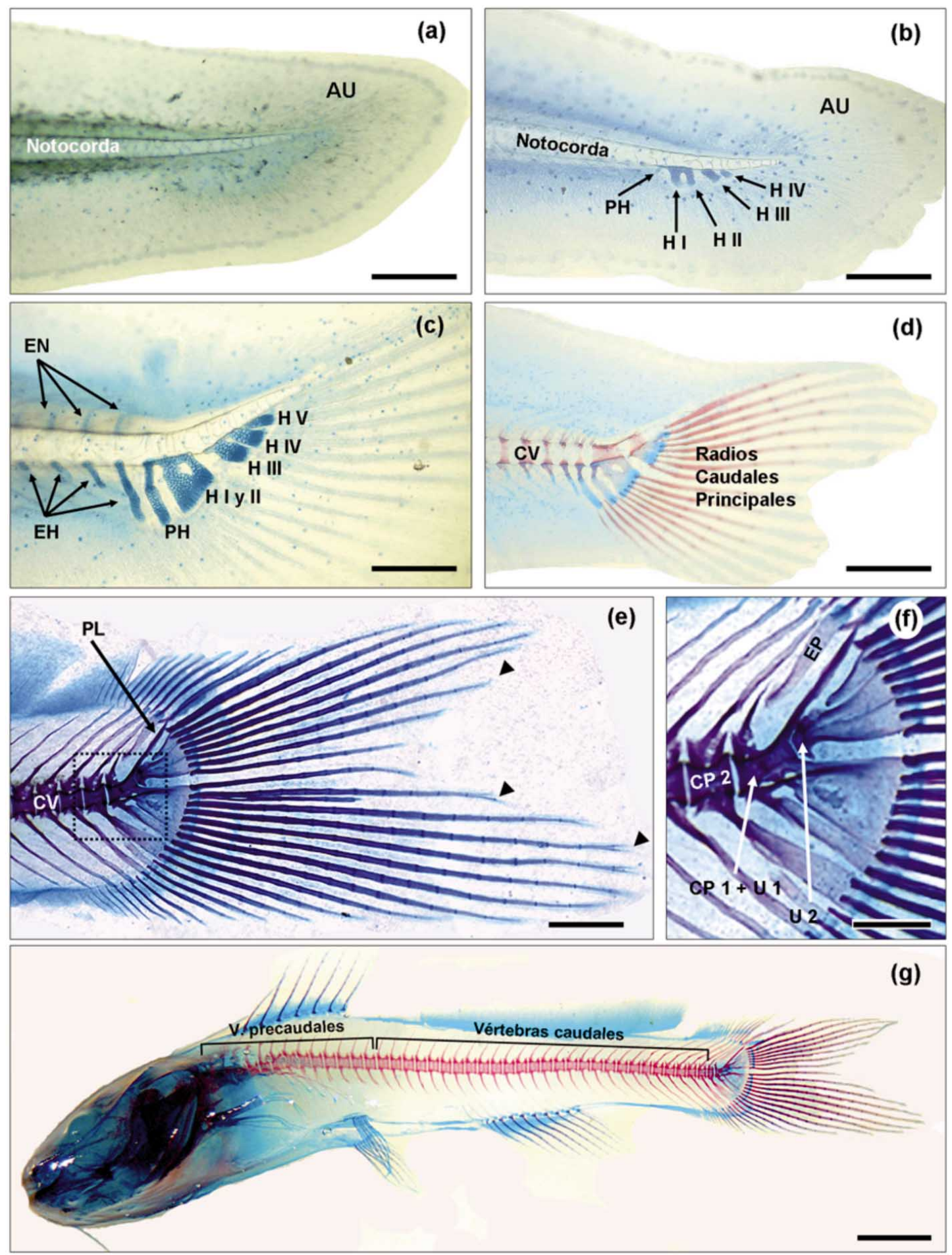

Fig. 1. Etapas del desarrollo de la columna vertebral y del esqueleto caudal en larvas de Rhamdia quelen. (a) Larva en preflexión (3 dpe). (b) Larva en flexión (4-5 dpe). (c) Larva en flexión, formación del esqueleto caudal (10-13 dpe). (d) Larva en flexión, osificación de los radios caudales y cuerpos vertebrales (15 dpe). (e) Larva en posflexión, esqueleto caudal completamente desarrollado 20 dpe, la cabeza de flecha (?) señala radios caudales principales ramificados. (f) Magnificación del recuadro de figura (e) donde se observa a los componentes del CCU. (g) Larva de 20 dpe. AU: pliegue de la aleta única, CP: centro preural, CV: cuerpos vertebrales, EH: espina hemal, EN: espina neural, EP: epural, H: hipural (I - V), N: notocorda, PH: parahipural, PL: pleurostilo U: ural. Barras: $(\mathrm{a}-\mathrm{b}-\mathrm{c})=250$ $\mu \mathrm{m} ;(\mathrm{d})=500 \mu \mathrm{m} ;(\mathrm{e})=1 \mathrm{~mm} ;(\mathrm{f})=500 \mu \mathrm{m} ;(\mathrm{g})=1,5 \mathrm{~mm}$. 


\section{DISCUSIÓN}

Los datos del presente trabajo complementan la información previa disponible sobre el desarrollo embrionario de R. quelen (Cussac et al., 1985; De Amorim et al., 2009), aunque ninguno de estos estudios hace referencia a las instancias de condrificación u osificación.

$\mathrm{Al}$ nacimiento, los peces presentan una gran variabilidad de estados de desarrollo osteológico (Koumoundouros et al., 2001; Gracian-Negrete et al., 2012). Parte de esta variabilidad depende de la cantidad de vitelo (Kendall et al., 1984), así como del tiempo o temperatura de incubación (Koumoundouros et al., 1997). En algunas especies, por ejemplo en Salmónidos, el desarrollo osteológico inicia seguido a la eclosión (Kendall \& Behnke, 1984), mientras que en la mayoría de las especies, el desarrollo esquelético inicia varios días después (Coban et al.; Gavaia et al.; Yildirim et al., 2009).

Según Fraser et al. (2004), el uso de larvas provenientes de piscicultura con el fin de describir el desarrollo esquelético puede incurrir en alteraciones morfológicas que no se ven en ejemplares silvestres. Sin embargo, este método permite obtener una muestra suficiente para la descripción ontogenética completa y por lo tanto es ampliamente utilizado (Grande \& Shardo, 2002; Coban et al.; Yildirim et al.).

La flexión de la notocorda da paso a la completa formación del esqueleto caudal (Kendall et al.). En el presente estudio este proceso se inició a los 4 dpe y finalizó a los 15 dpe con la completa formación del esqueleto caudal, constituido por cinco hipurales (H 1-5), dos centro urales (CU 1 y 2 ), un centro preural (CPU), un pleurostilo y un epural, hallándose además el $\mathrm{H} 1$ y 2 fusionados. Esta observación es similar a lo descrito en Helogenes marmoratus por Lundberg $\&$ Baskin (1969). Por el contrario, en Ictalurus punctatus se describen seis hipurales, estando los $\mathrm{H} 1$ y 2 separados, mientras que el 3 y 4 son los que se hallan fusionados (Grande \& Shardo).

En el presente estudio observamos un elemento epural independiente que corresponde con lo descrito anteriormente por Lundberg \& Baskin. El esqueleto caudal de R. quelen corresponde al tipo denominado pleurostilar (Patterson, 1968), donde el uroneural se fusiona con el centro preural $1 \mathrm{y}$ ural 1 para constituir el pleurostilo (Monod, 1968). Si bien estas estructuras se encuentran fusionadas en I. punctatus, los autores lo mencionan como uroneural (Grande \& Shardo).

Por su parte, los cuerpos vertebrales así como las espinas neurales y hemales inician su desarrollo pocos días después del nacimiento, posteriormente al inicio de la formación del esqueleto caudal, tal como fue observado en I. punctatus (Grande \& Shardo).

En ambientes naturales, una vez que las larvas agotan sus reservas nutricionales provenientes del vitelo deben obtener el alimento del medio externo. La búsqueda de alimentos, así como evitar ser depredadas supone el desarrollo de la capacidad locomotora (Koumoundouros et al., 2001). La fuerza de propulsión en la parte caudal del cuerpo aumenta como resultado del desarrollo de la aleta caudal (Matsuoka, 1987), permitiendo llevar a cabo funciones vitales para la supervivencia y la alimentación de las larvas en los primeros estadíos de desarrollo.

Concordando con Kuzir et al. (2009) este estudio contribuye al conocimiento del desarrollo ontogenético de $R$. quelen y además sienta la base para futuros estudios de malformaciones esqueléticas asociadas a los sistemas de larvicultura y a la nutrición. La detección precoz de malformaciones y la eliminación de las camadas de peces criados con una alta incidencia de este tipo de patologías, necesita de un conocimiento detallado del desarrollo normal en larvas (Koumoundouros et al., 1997). Además, el presente trabajo provee información para estudios de filogenia y clasificación del género Rhamdia, ya que se describe por primera vez el desarrollo osteológico de la columna vertebral y del esqueleto caudal en larvas de Rhamdia quelen bajo condiciones de laboratorio y con alimentación artificial. El desarrollo de la columna vertebral y del esqueleto caudal muestra patrones similares a los descriptos en otros peces teleósteos.

HERNÁNDEZ, D. R.; CASCIOTTA, J. R.; SANTINÓN, J. J.; SÁNCHEZ, S. \& DOMITROVIC, H.A. Ontogenic development of the vertebral column and caudal skeleton in Rhamdia quelen larvae in larviculture intensive. Int. J. Morphol., 30(4):1520-1525, 2012.

SUMMARY: The osteological development of vertebral column and caudal skeleton in Rhamdia quelen larvae under controlled conditions and artificial feeding is analyzed. Between 1 to 20 day post hatching (dph) four hundred larvae were sampled at regular intervals, and subsequently colored and transparent following the Taylor \& Van Dyke technique. In the first days of life, larvae have a straight notochord throughout its length. The beginning of the vertebral column formation was observed at 7 dph with the emergence of neural and haemal spines arches, and between 9 and $10 \mathrm{dph}$ for the first three vertebrae. The ossification of the vertebral column occurred in cranial-caudal direction and was complete at $20 \mathrm{dph}$. The caudal skeleton begins to develop between 3 and $4 \mathrm{dph}$, with the formation of the hypurals $(\mathrm{H})$, 
parahipural $(\mathrm{PH})$ and caudal fin rays. The $\mathrm{H} 1$ to 4 were formed as cartilaginous primordia on the ventral surface of the distal portion of the notochord, and then later the $\mathrm{H} 5$ and $\mathrm{PH}$ in distal and proximal extreme of the caudal skeleton, respectively. Central ural complex (CUC) was ossified in two parts, one anterior that is a combination of central preural 1 and ural 1 and other posterior with the ural 2. The first caudal fin rays appear in flexion larvae, while after $13 \mathrm{dph}$, all specimens had ossified principal rays (eight rays in both upper and lower lobes). The development of the vertebral column and caudal skeleton shows similar patterns to those described in other teleost fish although with some peculiarities that differentiate it from related groups.

KEY WORDS: Rhamdia quelen; Larviculture; Osteological development.

\section{REFERENCIAS BIBLIOGRÁFICAS}

Baldisserotto, B. \& Radünz Neto, J. Criação de jundiá. Santa Maria, UFSM, 2004. p.232.

Cahu, C.; Zambonino Infante, J. L. \& Takeuchi, T. Nutritional components affecting skeletal development in fish larvae. Aquaculture, 227(1-4):245-58, 2003.

Castro, J.; Pino-Querido, A.; Hermida, M.; Chavarrías, D.; Romero, R.; García-Cortés, L. A.; Toro, M. A. \& Martínez, P. Heritability of skeleton abnormalities (lordosis, lack of operculum) in gilthead seabream (Sparus aurata) supported by microsatellite family data. Aquaculture, 279(1-2):18-22, 2008. http://www.sciencedirect.com/science/article/pii/ S0044848608002998?_fmt $=$ full\&_origin $=\& \mathrm{md} 5=$ 7bcf780d0ad55e08c858017474822c86

Cussac, V. E.; Matkovic, M. \& Maggese, M. C. Desarrollo embrionario de Rhamdia sapo (Valenciennes, 1840) Eigenmann y Eigenmann, 1888 (Pisces, Pimelodidae). 2. Organogénesis media, organogénesis tardía y eclosión. Rev. Bras. Biol. 45(12):149-60, 1985.

Coban, D.; Suzer, C.; Kamaci, H. O.; Saka, S. \& Firat, K. Early osteological development of the fins in the hatchery-reared red porgy, Pagrus pagrus (L. 1758). J. Appl. Ichthyol., 25:26-32, 2009.

De Amorim, M. P.; Campos Gomes, B. V.; Martins, Y. S.; Sato, Y.; Rizzo, E. \& Bazzoli, N. Early development of the silver catfish Rhamdia quelen (Quoy \& Gaimard, 1824) (Pisces: Heptapteridae) from the São Francisco River Basin, Brazil. Aquaculture Res., 40(2):172-80, 2009.

Dunn, J. R. The utility of developmental osteology in taxonomic and systematic studies of teleost larvae: a review. NOAA Technical Report NMFS Circ 450. Rockville, U.S. Dept. of Commerce, National Oceanic and Atmospheric Administration, National Marine Fisheries Service, 1983. pp.1-19.
Fraser, M. R.; Anderson, T. A. \& de Nys, R. Ontogenic development of the spine and spinal deformities in larval barramundi (Lates calcarifer) culture. Aquaculture, 242(14):697-711, 2004.

Gracian-Negrete, J. M.; González-Acosta, A. F.; González-Isáis, M.; Ortiz-Galindo, J. L. \& Del Moral-Flores, L. F. Comparative osteology of the caudal skeleton of Achirus lineatus and Achirus mazatlanus (Pleuronectiformes: Achiridae). Int. J. Morphol., 30(2):705-8, 2012.

Gavaia, P. J.; Dinis, M. T. \& Cancela M. L. Osteological development and abnormalities of the vertebral column and caudal skeleton in larval and juvenile stages of hatcheryreared Senegal sole (Solea senegalensis). Aquaculture, 211(14):305-23, 2002.

Grande, T. \& Shardo, J. D. Morphology and development of the postcranial skeleton in the channel catfish Ictalurus punctatus (Ostariophysi: Siluriformes). Fieldiana Zool., 99:1-30, 2002.

Hernández, D. R.; Sánchez, S.; Santinón, J. J. \& Domitrovic, H. A. Fontes não-convencionais de proteína na primeira alimentação do bagre sul americano (Rhamdia quelen). Cienc. Rural, 39(3):878-84, 2009.

Kendall, A. W. \& Behnke, R. J. Salmonidae: Development and Relationships. In: Moser, H. G.; Richards, W. J.; Cohen, D. M.; Fahay, M. P. Kendall Jr. A. W. \& Richardson, S. L. (Eds.). Ontogeny and Systematics of Fishes. American Society of Ichthyologists and Herpetologists, Special Publication no 1. Lawrence, KS, Allen Press, 1984. pp.142-9.

Kendall, A. W.; Ahlstrom, E. H. \& Moser, H. G. Early life history stages of fishes and their characters. In: Moser, H. G.; Richards, W. J.; Cohen, D. M.; Fahay, M. P. Kendall Jr. A. W. \& Richardson, S. L. (Eds.). Ontogeny and Systematics of Fishes. American Society of Ichthyologists and Herpetologists, Special Publication no 1 Lawrence, KS, Allen Press, 1984. pp.11-22.

Koumoundouros, G.; Gagliardi, F.; Divanach, P.; Boglione, C.; Cataudella, S. \& Kentouri, M. Normal and abnormal osteological development of caudal fin in Sparus aurata L. fry. Aquaculture, 149(3-4):215-26, 1997.

Koumoundouros, G.; Divanach, P. \& Kentouri, M. Osteological development of Dentex dentex (Osteichthyes: Sparidae): Dorsal, anal, paired fins and squamation. Mar. Biol., 138(2):399406, 2001.

Lall, S. P. \& Lewis-McCrea, L. M. Role of nutrients in skeletal metabolism and pathology in fish - An overview. Aquaculture, 267(1-4):3-19, 2007.

Luchini, L. \& Panné Huidobro, S. Perspectivas en acuicultura: nivel mundial, regional y local. Buenos Aires, Dirección de Acuicultura -Subsecretaría de Pesca y Acuicultura, 2008. p.99. 
HERNÁNDEZ, D. R.; CASCIOTTA, J. R.; SANTINóN, J. J.; SÁNCHEZ, S. \& DOMITROVIC, H. A. Desarrollo ontogénico de la columna vertebral y del esqueleto caudal en larvas de Rhamdia quelen en condiciones de larvicultura intensiva. Int. J. Morphol., 30(4):1520-1525, 2012.

Lundberg, J. G. \& Baskin, J. N. The caudal skeleton of the catfishes, order Siluriformes. Am. Mus. Novit., 2398:1-49, 1969.

Matsuoka, M. Development of the skeletal tissues and skeletal muscles in the red sea bream. Bull. Seikai reg. Fish. Res. Lab., 65:1-114, 1987.

Monod, T. Le complexe urophore des poissons téléostéens. Mém. Inst. Fr. Afr. Noire, 81:1-705, 1968.

Patterson, C. The caudal skeleton on lower liassic pholidophorid fishes. Bull. Br. Mus. Nat. Hist., 16:203-39, 1968.

Kuzir, S.; Kozaric, Z.; Gjurcevic, E.; Bazdaric, B. \& Petrinec, Z. Osteological development of the garfish (Belone belone) larvae. Anat. Histol. Embryol., 38(5):351-4, 2009.

Sfakianakis, D. G.; Georgakopoulou, E.; Papadakis, I.; Divanach, P.; Kentouri, M. \& Koumoundouros, G. Environmental determinants of hemal lordosis in European sea bass, Dicentrarchus labrax (Linnaeus, 1758). Aquaculture, 254:5464, 2006

Silveira, F. \& Müller F. Síntese informativa da atividade de piscicultura de água doce 2010. da Epagri, Centro de Desenvolvimento em Aqüicultura e Pesca (Cedap), 2010. p.99.

Yildirim, S.; Coban, D.; Kamaci, O. H.; Süzer, C.; Saka, S. \& Firat, $\mathrm{K}$. Skeletal formation of the fins in the common dentex (Dentex dentex L., 1758) under intensive culture condition. J. Fish Aquat. Sci., 26(3):165-70, 2009.

\section{Dirección para correspondencia: David Roque Hernández Instituto de Ictiología del Nordeste Facultad de Ciencias Veterinarias Universidad Nacional del Nordeste ARGENTINA}

Email: dhernandez@vet.unne.edu.ar

Recibido : 11-04-2012

Aceptado: 05-06-2012 